\title{
Waterpipe (hookah) tobacco use in pregnancy: use, preferences and perceptions of flavours
}

\author{
Laura Stroud (1) 1,2 Erika Werner, ${ }^{3,4}$ Kristen Matteson, $^{3,4}$ Michael Carey, ${ }^{1,2}$ \\ Gideon St Helen, ${ }^{5}$ Thomas Eissenberg, ${ }^{6}$ Lori A J Scott-Sheldon ${ }^{1,2}$
}

\begin{abstract}
${ }^{1}$ Psychiatry and Human Behavior, Brown University Warren Alpert Medical School, Providence, Rhode Island, USA ${ }^{2}$ Centers for Behavioral and Preventive Medicine, The Miriam Hospital, Providence, Rhode Island, USA

${ }^{3}$ Obstetrics and Gynecology, Brown University Warren Alpert Medical School, Providence, Rhode Island, USA

'Women \& Infants' Hospital of Rhode Island, Providence, Rhode Island, USA

${ }^{5}$ Division of Clinical

Pharmacology, Department of Medicine, University of California, San Francisco, San Francisco, California, USA ${ }^{6}$ Center for the Study of Tobacco Products, Department of Psychology, Virginia Commonwealth University, Richmond, Virginia, USA
\end{abstract}

\section{Correspondence to} Professor Laura Stroud and Professor Lori A J Scott-Sheldon, Centers for Behavioral and Preventive Medicine, The Miriam Hospital, Providence, Rhode Island, USA;

Laura_Stroud@brown.edu, Lori_Scott-Sheldon@brown.edu

Received 30 January 2019 Revised 10 May 2019 Accepted 13 May 2019 Published Online First 18 July 2019
ABSTRACT

Objective Waterpipe tobacco (WPT; hookah) use is common in pregnant and reproductive-age women. Sweet flavours contribute to the appeal of WPT and are a potential regulatory target. This study investigated use, preferences and perceptions of WPT flavours in pregnant WPT users, and the impact of flavour preferences on preconception/prenatal WPT use and exposure biomarkers.

Methods 58 pregnant WPT users (mean age $=27$ years) completed a detailed interview regarding their WPT

flavours use, preferences and perceptions. Biomarkers of nicotine and carcinogen exposure (eg, cotinine, benzene, butadiene) were also collected.

Results $55 \%$ of participants were dual/poly WPT users (ie, reported use of one or more other tobacco products in addition to WPT). Pregnant WPT users reported nearly exclusive use of flavoured WPT, with greater use of menthol/mint (68\%) followed by fruit flavours (48\%) $(p<0.001)$, and greater preferences for fruit followed by menthol/mint flavours (ps<0.05). Harm perceptions did not differ among flavours. Compared with dual/poly WPT users, WPT-only users reported more total WPT use events, greater use of and preference for menthol/mint flavoured WPT (ps<0.001), and decreased exposure biomarkers ( $p s \leq 0.040$ ). Preference for menthol/mint and fruit flavours predicted more flavoured WPT use events during preconception and pregnancy; preference for menthol/mint predicted detectable cotinine and benzene levels but not butadiene.

Conclusions This is the first study of WPT flavour use, preferences and perceptions in pregnant women. Use of and preference for menthol/mint and fruit WPT flavours in this vulnerable population could be considered in regulating WPT flavours to protect the health of women and children.

\section{INTRODUCTION}

Waterpipe (hookah) tobacco (WPT) smoking is a centuries-old tradition that evolved in the Middle East and India but has become an increasing public health problem in the USA and worldwide. While cigarette use has declined significantly in the USA, ${ }^{1}$ use of other tobacco products including WPT is increasing, particularly among young adults. ${ }^{2-7}$ There is also evidence for increasing WPT use and social acceptability among reproductive-age women. $^{8-11}$ However, despite well-known causal effects of tobacco use in pregnancy on maternal, fetal and infant morbidity, only a small number of studies have addressed WPT use in pregnant women, a uniquely vulnerable population. ${ }^{1}{ }^{12}$ In several studies from Lebanon, Iran and Jordan, the prevalence of WPT use in pregnancy ranged from $5.4 \%$ to $8.7 \% .^{13-18}$ In the Population Assessment of Tobacco and Health (PATH) study in the USA, WPT was the third most prevalent tobacco product used by pregnant women after cigarettes and e-cigarettes. ${ }^{11}$ Prevalence of WPT use in pregnancy was $2.5 \%$ in the overall sample but $12.4 \%$ among pregnant cigarette smokers.

Because WPT involves burning charcoal to heat (flavoured) tobacco, WPT users are exposed to toxic combustion products as well as nicotine and other toxicants. ${ }^{19}$ In laboratory studies, relative to a single cigarette, WPT smokers were exposed to 1.7 times the nicotine, 3.7 times the carbon monoxide (CO) and 50 times the smoke. ${ }^{20}{ }^{21}$ WPT use is also associated with exposure to volatile organic compounds (VOCs), including 1,3-butadiene, acrylonitrile, acetaldehyde and benzene, that have the highest cancer risk indices. ${ }^{22}$ WPT use (vs cigarettes) is associated with particular increases in benzene, ${ }^{23} 24$ a carcinogen linked to leukaemia, the most common cancer in children and teens. ${ }^{25}$ Finally, WPT use is associated with health outcomes similar to those associated with cigarettes, including respiratory disease and oral and lung cancer. ${ }^{26-29}$

The issue of health risks and toxicity from WPT use is even more salient among pregnant women due to impact of maternal use on both mother and the developing fetus. Decades of research have supported causal links between maternal prenatal cigarette use and obstetric complications (eg, premature delivery, placental abruption), as well as infant morbidity and mortality (eg, low birthweight, sudden infant death syndrome). ${ }^{112}$ A small number of studies have investigated WPT use and health outcomes among pregnant women in the Middle East (eg, Lebanon, Iran, Jordan) and Southeast Asia (eg, Cambodia). ${ }^{16}$ 29-31 WPT-exposed infants were 3.7 times more likely to have respiratory problems and were 2.4 times more likely to be born low birthweight (weight less than $2500 \mathrm{~g}$ ) than unexposed infants. ${ }^{26}$ Low birthweight infants are at increased risk for admittance to neonatal intensive care and for poorer long-term outcomes. ${ }^{32}$ WPT-exposed children were also at increased risk for asthma. ${ }^{33}$

Despite adverse health outcomes of WPT use, it is often perceived as less harmful than cigarette use. ${ }^{34}$ Decreased harm perceptions and increased social acceptability of WPT may be due to the social and more sporadic nature of its use, flavourings and misperception that harmful toxicants from tobacco are 'filtered' by the water. ${ }^{34-36}$ In addition, there is a dearth of regulations and poor compliance with existing regulations to control the use and spread of 
WPT. ${ }^{37}$ A review of tobacco control legislation in 62 countries found that WPT-specific language was absent from such legislation in the majority of countries. ${ }^{37}$ In the USA, the Food and Drug Administration asserted regulatory authority over WPT in $2016 .{ }^{38}$ To facilitate the development of a WPT-specific regulatory framework to protect vulnerable populations such as pregnant women, it is critical to identify WPT characteristics that are associated with higher use and are amenable to regulation.

One reason for the increasing global appeal of WPT is the introduction of maassel, a sweetened, flavoured tobacco product made up of shredded tobacco, glycerol, flavours (eg, candy, fruit) and other additives. ${ }^{9}{ }^{40}$ Maassel simplified the WPT preparation process-allowing for increased availability, flavour variety and palatability of WPT. Thus, maassel and WPT flavours represent an important potential regulatory target. Pregnant women may be especially vulnerable to the appeal of WPT flavours due to alterations in taste, cravings and nausea during pregnancy. ${ }^{41}$ We recently showed higher use of and preference for fruit and menthol/mint versus unflavoured e-cigarettes in pregnancy and preconception, and links between preferences for flavoured e-cigarettes and lifetime use of e-cigarettes by pregnant women. ${ }^{42}$ We also showed higher preference for sweet WPT flavours in reproductive-age women. ${ }^{43}$

In the present study, we extend this work to investigate use, preferences and perceptions of WPT flavours in pregnant users, including WPT-only users and WPT users who use other tobacco products (dual/poly WPT users). Our aims were to investigate: (1) flavoured WPT use over preconception and first trimester in pregnant WPT-only and dual/poly WPT users, (2) preferences for and perceptions of WPT flavours in pregnant WPT-only and dual/poly users and (3) the impact of flavour preferences on use and biomarkers of exposure, including nicotine, CO and VOCs. Based on our prior work, ${ }^{42} 43$ we hypothesised that fruit, sweet and menthol/mint flavours would be used most frequently and preferred by pregnant WPT users. Further, we hypothesised that preferences for sweet and menthol/mint flavours would be associated with greater use of WPT and dual/poly WPT use and higher levels of biomarkers of exposure.

\section{METHODS}

\section{Overview}

The present study involved a cross-sectional analysis of baseline data from the first cohort of an ongoing longitudinal study of pregnant WPT users. This cohort included 58 pregnant WPT users (26 WPT-only, 32 dual/poly WPT users). Participants completed a baseline interview and biomarker collection session in early pregnancy. To minimise potential biases, (1) study interviews and data analyses were conducted by different individuals, (2) interview staff received training based on project-specific protocols, (3) $36 \%$ of audiotapes from the baseline interview were reviewed for quality control and (4) biomarkers of use/ exposure were used in addition to maternal self-report data. All participants were compensated US $\$ 50$ for their participation in the baseline session; 36 of the 58 participants (62\%) received a US $\$ 50$ bonus for keeping their scheduled appointment.

\section{Screening and recruitment}

Pregnant women responded to invitations (face-to-face contact, brochures, paper and electronic advertisements) to the 'Let's Talk about Hookah' study offered at multiple obstetric offices and community sites within Rhode Island. Although this was a convenience sample of 58 pregnant WPT users in southeastern New England (98\% residents of Rhode Island; 2\%
Massachusetts), recruitment sites were chosen to cover a breadth of pregnant women and to reach women of diverse racial/ ethnic identities and a range of socioeconomic status. Face-toface recruitment was conducted by research staff not involved in prenatal care. One hundred and four women who expressed interest in the study were screened by telephone between August 2017 and August 2018 to determine whether they met the eligibility criteria: (1) English-speaking, (2) aged 18-40 years, (3) WPT use at least once during pregnancy or preconception (ie, 3 months prior to conception), (4) a singleton pregnancy and (5) no severe medical (pre-eclampsia) or psychiatric (bipolar or psychotic disorder) conditions during pregnancy. Nine women were no longer interested when called and 14 were ineligible; 21 women did not attend their baseline interview and could not be rescheduled, leaving 60 participants who consented and enrolled. Two participants were excluded following the baseline interview due to no WPT use during preconception/pregnancy and a bipolar diagnosis. Of the 58 participants, 56 were enrolled via face-to-face recruitment efforts in obstetric offices; two were enrolled from posted advertisements or friends.

\section{Interview session}

Participants completed the baseline interview session during the first or second trimester $(M=14, S D=4$ weeks gestation; range $=7-25)$. The interview assessed (1) demographic information (eg, age, ethnicity) and (2) general health, pregnancy and tobacco history. We used the timeline follow back (TLFB) method to assess (3) tobacco and other substance use ${ }^{44-46}$ and the tobacco flavours interview ${ }^{47}$ to assess (4) flavour preferences.

\section{Measures}

Timeline followback

The TLFB is a structured calendar-based assessment of daily substance use that uses key dates (eg, holidays, personal events) to cue memory and increase accuracy of recall. ${ }^{44-46}$ The TLFB was used to assess daily WPT use and other tobacco/nicotine product use (ie, cigarettes, e-cigarettes, cigars/cigarillos/ little cigars), alcohol and other substance use, and exposure to secondhand WPT and cigarette smoke. The TLFB interview covered each day of pregnancy up to the interview day and the 3 months prior to conception. For each episode of WPT use, mothers were asked about the brand, flavours, amount of use (ie, quantity and duration), and social context (eg, where and with whom) of their use. The data collected from the TLFB was used to determine (1) the number of WPT-only and dual/poly WPT users (How many times did you use (TOBACCO PRODUCT) in the past $\mathrm{x}$ months?) and (2) the number of flavour use events (Did you smoke hookah that was flavoured? If yes: What flavour or flavours did you smoke?). Participants who reported use of any non-WPT tobacco product (ie, cigarettes, e-cigarettes, cigars/ cigarillos/little cigars) during preconception or pregnancy were categorised as dual/poly users. Participants who reported use of flavoured WPT during preconception or pregnancy were categorised as using any one of ten flavour categories (see below; eg, menthol/mint, fruit) during that WPT use event.

\section{Tobacco flavours interview: overview}

The tobacco flavours Interview involves the use of a portable flip chart presentation easel binder. ${ }^{47}$ The interview includes a series of questions regarding use, preferences, perceptions and intentions to use flavoured WPT across 10 flavour categories (menthol/mint, clove/spice, fruit, chocolate, alcohol (eg, margarita), coffee, or other beverages (eg, lemonade), candy or 
other sweets (eg, cotton candy, butterscotch) and unflavoured) consistent with the flavour categories from the PATH Study. ${ }^{48}$ The presentation easel binder includes photos of WPT products, flavour images (eg, fruit, alcohol) and response choices (eg, 1-7 scale with anchors of extremely dislike to extremely like).

\section{Tobacco flavours interview: measures}

All measures were pilot tested in a sample of 630 reproductiveaged women. ${ }^{43}$ Higher ratings indicate more positive preferences for and perceptions of harm for the specific flavour.

Preferences for WPT flavourings: Preferences for WPT flavours were measured using four items reported on a 7-point Likert scale (extremely dislike-extremely like, extremely unattractive-extremely attractive, extremely unpleasant-extremely pleasant, not at all interested-very interested).

General and pregnancy-specific risk perceptions of WPT: Participants were asked about their perceptions of general ('for your health') and pregnancy-related ('for pregnant women to use,' and 'to the fetus') risks across all WPT flavours using three items reported on a 7-point scale (not at all harmful-very harmful) adapted from published measures. ${ }^{49} 50$

\section{Biomarkers of WPT/tobacco smoke exposure}

Saliva cotinine is a reliable biomarker for nicotine exposure. ${ }^{51}$ Maternal saliva samples were collected at the interview session then frozen until analysis by University of California, San Francisco (UCSF) Clinical Pharmacology Research Laboratory using gas chromatography with nitrogen-phosphorus detection, modified for use with a capillary column. ${ }^{5253}$ Limit of quantification (LOQ) for cotinine was $10 \mathrm{ng} / \mathrm{mL}$.

Breath $\mathrm{CO}$ concentrations were measured using the Micro $+{ }^{\mathrm{TM}}$ basic Smokerlyzer (coVita, Santa Barbara, California, USA) as a quantitative measure of exposure to combustion products. ${ }^{54}$

Urine Mercapturic Acid Metabolites of VOCs: Maternal urine samples were collected at the interview session for determination of mercapturic acid metabolites of acrolein (3-hydroxypropylmercapturic acid, 3-HPMA), acrylamide (2-carbamoylethylmercapturic acid), acrylonitrile (2-cyanoethylmercapturic acid), benzene (phenylmercapturic acid), crotonaldehyde (3-hydroxy-1-methylpropylmercaptu ric acid), ethylene oxide (2-hydroxyethylmercapturic acid), propylene oxide (2-HPMA), 1,3-butadiene (sum of isomers 1-h ydroxy-3-buten-2-yl-mercapturic acid and 2-hydroxy-3-buten-1 -yl-mercapturic acid, MHBMA-1+2, and 4-hydroxy-2-buten-1yl-mercapturic acid, MHBMA-3) and methylating agents (methylmercapturic acid). Samples were frozen until analysis by UCSF using liquid chromatography with tandem mass spectrometry. ${ }^{23}$ LOQs ranged from 0.1 to $5 \mathrm{ng} / \mathrm{mL}$.

\section{Data management and statistical analysis}

All data analyses were conducted using Stata V.15. ${ }^{55}$ Differences in characteristics and biomarkers between WPT-only and dual/ poly users were examined using a series of bivariate analyses $(\chi 2$, t-tests) (table 1). Preferences and perceptions of WPT flavours were significantly skewed and therefore evaluated using KruskalWallis analysis of variance, followed by Dunn's tests with a Bonferroni adjustment. Differences between flavour preferences and perceptions by user type were assessed using a two-sample Wilcoxon-rank sum test (table 2). Because a ceiling effect was observed for the perceptions of pregnancy harm (67\% endorsed 'very harmful' for mint/menthol and fruit-flavours), this scale was omitted from all regression analyses but included in table 2 for descriptive purposes. Biomarker levels were positively skewed and were log transformed prior to descriptive analyses. Biomarker values below the limit of quantification (BLQ) were imputed using standard methods (BLQ/ $/ \sqrt{2}),{ }^{56}$ and those in urine were creatinine-normalised to adjust for differences in urine dilution. ${ }^{57}$

Regression analyses were used to determine the association between preferences, perceptions and user type $(0=\mathrm{WPT}$ only; $1=$ dual/poly WPT use) on total number of flavoured WPT use events during preconception or pregnancy. Based on prior research on non-cigarette tobacco use, ${ }^{58}$ age (continuous) and ethnicity ( $1=$ Latina; $0=$ non-Latina) were included as covariates. A number of menthol/mint and fruit-flavoured WPT use events were skewed and clustered at zero, with the variance surrounding the means indicating a high degree of overdispersion; therefore, negative binomial regression analysis with robust standard errors was used. Preferences (liking, attractiveness, pleasant, interest) exhibited a high degree of multicollinearity for menthol/mint (variance inflation factor (VIF) $\geq 8.20$ ) and fruit (VIF $\geq 3.79$ ) and were averaged to create a mean preference score.

Logistic regression analyses were used to predict biomarkers (ie, cotinine, benzene, butadiene) that were detectable in the sample $(1=$ yes; $0=\mathrm{BLQ})$ from preferences, perceptions general harm, harm to the fetus), and total number of flavoured WPT use events during preconception or pregnancy. (Age and ethnicity were included as covariates in these models.) Cotinine was selected as the primary proximate metabolite of nicotine intake ${ }^{59}$; benzene and butadiene were selected due to their association with WPT in particular and causal links with haematological malignancies. ${ }^{2425}{ }^{60}$ Missing biomarker data was omitted from analyses: $(\mathrm{N}=1$ for saliva cotinine and $\mathrm{CO} ; \mathrm{N}=27$ for urine VOCs).

\section{RESULTS}

\section{Sample characteristics}

Characteristics of the overall sample and stratified by WPT-only and dual/poly WPT users are provided in table 1 . The sample included 58 pregnant WPT users $\left(\mathrm{M}_{\text {age }}=27 ; 19-39\right.$ years; 72\% $(\mathrm{n}=42)$ non-white race; $53 \%(\mathrm{n}=31)$ Latino ethnicity; $83 \%$ unmarried; $50 \% \leq$ high school education). The sample was not representative of pregnant women in Rhode Island $\left(\mathrm{M}_{\mathrm{age}}=30\right.$; 15-45 years; $21 \%$ non-white race; $26 \%$ Latino; $44 \%$ unmarried; $33 \% \leq$ high school education). ${ }^{61}$ Dual/poly WPT users reported using cigarettes $(n=8 ; 25 \%$ of dual/poly users), e-cigarettes $(n=3 ; 9 \%)$, cigars $(n=9 ; 28 \%)$, cigarettes + cigars $(n=4 ; 13 \%)$, e-cigarettes + cigars $(n=1,3 \%)$ or cigarettes+e-cigarettes + cigars $(\mathrm{n}=7 ; 22 \%)$ during pregnancy or preconception. Dual/poly WPT users were less likely to identify as Latina ethnicity compared with WPT-only users (38\% vs $73 \%$ identified as Latina ethnicity, respectively). Dual/poly users were more likely to report marijuana use $(\mathrm{p}<0.001)$ and secondhand cigarette smoke exposure $(\mathrm{p}=0.030)$ versus WPT-only users.

\section{Biomarkers of nicotine intake, $\mathrm{CO}$ and VOCs}

Table 1 provides biomarkers of nicotine intake, CO concentration and carcinogen exposure for the overall sample by user type. Levels of biomarkers were low, with more than $>50 \%$ BLQ for cotinine, and mercapturic acid metabolites of benzene, butadiene and acronitrile; $84 \%$ of CO values were below the cut-off for active smoking $\left(\leq 3 \mathrm{ppm}^{62}\right)$. Dual/poly users showed increased levels of cotinine, acrylonitrile, acrolein, propyleneoxide and acrylamide versus WPT-only users (ps $\leq 0.040$ ). Dual/ poly users were also more likely to show positive $\mathrm{CO}$ readings $(26 \%(n=8 / 31)$ vs $4 \%(n=1 / 26)), \chi^{2}(1)=5.13, p=0.024$. 
Table 1 Sample characteristics and biomarker levels by WPT use $(\mathrm{N}=58)$

\begin{tabular}{|c|c|c|c|c|}
\hline & Overall $\mathrm{N}=58$ & WPT-only users $n=26$ & Dual/Poly users $n=32$ & \\
\hline & $\%(n)$ & $\%(\mathrm{n})$ & $\%(\mathrm{n})$ & $P$ value \\
\hline \multicolumn{5}{|l|}{ Maternal characteristics } \\
\hline Age (years), M (SD) & $27(5)$ & $27(5)$ & $27(5)$ & 0.636 \\
\hline Non-white race* & $72 \%(42)$ & $81 \%(21)$ & $66 \%(21)$ & 0.199 \\
\hline Latina ethnicity & $53 \%(31)$ & $73 \%(19)$ & $38 \%(12)$ & 0.007 \\
\hline$\leq$ High school education & $50 \%(29)$ & $38 \%(10)$ & $59 \%(19)$ & 0.113 \\
\hline Income < US $\$ 30$ 000/year & $38 \%(22)$ & $31 \%(8)$ & $44 \%(14)$ & 0.311 \\
\hline Unemployed & $40 \%(23)$ & $42 \%(11)$ & $38 \%(12)$ & 0.710 \\
\hline Unmarried & $83 \%(10)$ & $73 \%(19)$ & $91 \%(29)$ & 0.078 \\
\hline Unplanned pregnancy & $69 \%(40)$ & $69 \%(18)$ & $69 \%(22)$ & 0.969 \\
\hline Parity, M (SD) & $1(1)$ & $1(1)$ & $1(1)$ & 0.316 \\
\hline \multicolumn{5}{|c|}{ Pregnancy tobacco and substance use/exposure } \\
\hline Any cigarette & $33 \%(19)$ & $0 \%(0)$ & $59 \%(19)$ & NA \\
\hline Any e-cigarette & $19 \%(11)$ & $0 \%(0)$ & $34 \%(11)$ & NA \\
\hline Any cigar & $36 \%(21)$ & $0 \%(0)$ & $66 \%(21)$ & NA \\
\hline Any alcohol & $88 \%(51)$ & $88 \%(23)$ & $88 \%(28)$ & 0.911 \\
\hline Any marijuana & $52 \%(30)$ & $23 \%(6)$ & $75 \%(24)$ & $<0.001$ \\
\hline Secondhand WPT exposure & $67 \%(39)$ & $69 \%(18)$ & $66 \%(21)$ & 0.085 \\
\hline Secondhand smoke exposure & $47 \%(27)$ & $31 \%(8)$ & $59 \%(19)$ & 0.030 \\
\hline Biomarkers & $n=57$ & $\mathrm{n}_{\text {cotinine/CO }}=25 / 26$ & $\mathrm{n}_{\text {cotinine/CO }}=32 / 31$ & \\
\hline Saliva cotinine (GM, \% BLQ, ng/mL) & $1.52(77 \%)$ & $0.71(96 \%)$ & $2.77(63 \%)$ & 0.002 \\
\hline Breath CO (ppm, \% <3 ppm) & $1.82(84 \%)$ & $1.41(96 \%)$ & $2.26(74 \%)$ & 0.092 \\
\hline Urine VOCs (GM, \% BLQ, ng/mg) & $\underline{n=31}$ & $\underline{n=16}$ & $\underline{n=15}$ & \\
\hline Benzene (PMA) & $0.12(61 \%)$ & $0.09(69 \%)$ & $0.14(53 \%)$ & 0.224 \\
\hline Butadiene (MHBMA 1+2,-3) & $0.08(68 \%)$ & $0.08(63 \%)$ & $0.07(73 \%)$ & 0.501 \\
\hline Ethylene oxide (HEMA) & $1.46(6 \%)$ & $1.06(6 \%)$ & $2.1(0 \%)$ & 0.075 \\
\hline Methylating agents (MMA) & $11.68(16 \%)$ & $8.84(25 \%)$ & $15.7(7 \%)$ & 0.218 \\
\hline Acrylonitrile (CNEMA) & $1.52(55 \%)$ & $0.52(69 \%)$ & $4.8(40 \%)$ & 0.007 \\
\hline Acrolein (3-HPMA) & $231.25(0 \%)$ & $175.3(0 \%)$ & $310.81(0 \%)$ & 0.040 \\
\hline Propylene oxide (2-HPMA) & $21.63(3 \%)$ & $15.4(6 \%)$ & $31.11(0 \%)$ & 0.039 \\
\hline Acrylamide (AAMA) & $54.76(0 \%)$ & $41.1(0 \%)$ & $74.33(0 \%)$ & 0.033 \\
\hline Crotonaldehyde (HPMMA) & $132.98(0 \%)$ & $115.5(0 \%)$ & $154.63(0 \%)$ & 0.191 \\
\hline
\end{tabular}

$\mathrm{N}=31$ for VOCs ( $\mathrm{N}=16$ WPT-only users; $\mathrm{N}=15$ dual/poly users). Bold values indicate statistically significant differences between WPT-only and dual/poly WPT users.

${ }^{*}$ Non-white race includes $21 \%(n=12)$ African American, 1.5\% $(n=1)$ Asian, 1.5\% ( $\left.n=1\right)$ American Indian/Alaska Native, 38\% ( $\left.n=22\right)$ other and 10\% ( $\left.n=6\right)$ mixed/unknown. AAMA, 2-carbamoylethylmercapturic acid; BLQ, below the limit of quantification for theassay; CNEMA, 2-cyanoethylmercapturic acid; CO, carbon monoxide; GM, geometric mean; HEMA, 2-hydroxyethylmercapturic acid; 3-HPMA, 3-hydroxypropylmercapturic acid; MHBMA 1+2, -3, 1-hydroxy-3-buten-2-yl-mercapturic acid and 2-hydroxy-3-buten1-yl-mercapturic acid, and 4-hydroxy-2-buten-1-yl-mercapturic acid; MMA, methylmercapturic acid; PMA, phenylmercapturic acid; VOC, volatile organic compounds; WPT, waterpipe tobacco.

\section{Prevalence and patterns of WPT use in preconception and first trimester}

Prevalence of WPT use was $81 \%(\mathrm{~N}=47)$ during pregnancy and $98 \%(\mathrm{~N}=57)$ during preconception. Participants reported using WPT an average of 8 times $(\mathrm{SD}=13$; range $=0-69)$ during first trimester and 15 times $(\mathrm{SD}=28$; range $=0-167)$ during preconception. On average, 26\% used WPT weekly, 22\% used $<$ weekly but $\geq$ monthly, and $52 \%$ used $<$ monthly. Sixty-eight per cent of pregnant WPT users reported using menthol/mint flavours, $48 \%$ reported using fruit flavours, 33\% reported using mint + fruit and 5\% reported using candy flavours. Figure 1 shows the proportion of flavours used across all WPT use events from the TLFB. Of the 1376 WPT use events (904 preconception/472 first trimester), 68\% included menthol/flavoured WPT, $14 \%$ fruit, $14 \%$ mint + fruit, $2 \%$ mint + coffee and $1 \%$ other flavours (ie, candy, fruit + candy, mint + chocolate, fruit + chocolate, coffee, alcohol, other beverages). Patterns were similar during preconception and first trimester. Marijuana was mixed with WPT in 59 (4\%) of events. WPT-only users reported more preconception $(M=24, S D=8$ vs $M=9, S D=16 ; p=0.024)$ and first trimester WPT use events $(\mathrm{M}=11, \mathrm{SD}=17$ vs $\mathrm{M}=6, \mathrm{SD}=8$; $\mathrm{p}=0.011)$ compared with dual/poly users. WPT-only users also reported more events using menthol/mint flavours compared with dual/poly users $(\mathrm{M}=30, \mathrm{SD}=55$ vs $\mathrm{M}=5, \mathrm{SD}=12$; $\mathrm{p}<0.001)$. There were no significant differences between WPTonly and dual/poly users for events with fruit flavours $(M=3$, $\mathrm{SD}=7$ vs $\mathrm{M}=4, \mathrm{SD}=10 ; \mathrm{p}=0.366)$ or use of WPT mixed with marijuana $(\mathrm{M}=0.04, \mathrm{SD}=0.20$ vs $\mathrm{M}=2, \mathrm{SD}=9 ; \mathrm{p}=0.178)$.

\section{Preferences and perceptions of flavoured WPT}

Table 2 documents preference and perceptions for each WPT flavour for the overall sample and stratified by WPT-only and dual/poly users. Mean preferences were significantly different between flavours for each preference scale (liking, attractiveness, pleasant, interest), ps<0.001). Fruit and menthol/mint were most preferred, followed by candy and other sweets; tobacco, spice and coffee were least preferred. Post hoc tests indicated that fruit preferences were higher $(\mathrm{ps}<0.05)$ than all other flavours except menthol/mint; menthol/mint preferences were 
Table 2 Mean flavour preferences and perceptions by WPT use among pregnant women ( $\mathrm{N}=58)$

\begin{tabular}{|c|c|c|c|c|c|c|c|c|c|c|}
\hline & Fruit & Menthol/Mint & Candy & Chocolate & Other Sweets & Alcohol & Coffee & Other beverages & Spice & Tobacco \\
\hline \multicolumn{11}{|l|}{ Preferences } \\
\hline \multicolumn{11}{|l|}{ Liking } \\
\hline All & 5.52 & 5.00 & 3.75 & 2.69 & 3.45 & 2.86 & 2.07 & 2.83 & 2.24 & 2.19 \\
\hline WPT-only & 5.31 & $5.62 *$ & 3.31 & 2.38 & 3.00 & 2.92 & 1.73 & 2.77 & 2.38 & 2.19 \\
\hline Dual/poly & 5.69 & $4.50^{*}$ & 4.09 & 2.94 & 3.81 & 2.81 & 2.34 & 2.88 & 2.13 & 2.19 \\
\hline \multicolumn{11}{|l|}{ Attractiveness } \\
\hline All & 5.09 & 4.66 & 3.55 & 2.62 & 3.31 & 2.78 & 2.14 & 2.88 & 2.21 & 2.16 \\
\hline WPT-only & 4.88 & $5.38^{*}$ & 3.31 & 2.35 & 2.81 & 2.88 & 1.77 & 2.81 & 2.31 & 2.35 \\
\hline Dual/poly & 5.25 & $4.06^{*}$ & 3.75 & 2.84 & 3.72 & 2.69 & 2.44 & 2.94 & 2.13 & 2.00 \\
\hline \multicolumn{11}{|l|}{ Pleasant } \\
\hline All & 5.03 & 4.78 & 3.59 & 2.59 & 3.31 & 2.81 & 2.05 & 3.00 & 2.16 & 2.03 \\
\hline WPT-only & 4.81 & $5.42 *$ & 3.19 & 2.19 & 2.73 & 2.77 & 1.65 & 2.81 & 2.35 & 2.23 \\
\hline Dual/poly & 5.22 & $4.25^{*}$ & 3.91 & 2.91 & 3.78 & 2.84 & 2.38 & 3.16 & 2.00 & 1.88 \\
\hline \multicolumn{11}{|l|}{ Interest } \\
\hline All & 4.93 & 4.74 & 3.53 & 2.48 & 3.26 & 2.62 & 1.93 & 2.91 & 2.07 & 1.98 \\
\hline WPT-only & 4.65 & $5.38^{*}$ & 3.19 & 2.00 & 2.92 & 2.65 & 1.65 & 2.88 & 2.15 & 2.12 \\
\hline Dual/poly & 5.16 & $4.22 *$ & 3.81 & 2.88 & 3.53 & 2.59 & 2.16 & 2.94 & 2.00 & 1.88 \\
\hline \multicolumn{11}{|l|}{ Perceptions } \\
\hline \multicolumn{11}{|l|}{ General harm } \\
\hline All & 4.91 & 5.03 & 4.91 & 4.98 & 4.91 & 5.22 & 5.09 & 4.93 & 5.05 & 5.29 \\
\hline WPT-only & 4.85 & 4.85 & 4.92 & 5.12 & 4.92 & 5.19 & 5.19 & 4.96 & 4.92 & 5.54 \\
\hline Dual/poly & 4.97 & 5.19 & 4.91 & 4.88 & 4.91 & 5.25 & 5.00 & 4.91 & 5.16 & 5.09 \\
\hline \multicolumn{11}{|c|}{ Pregnancy harm } \\
\hline All & 6.17 & 6.21 & 6.12 & 6.14 & 6.12 & 6.36 & 6.26 & 6.19 & 6.17 & 6.36 \\
\hline WPT-only & 6.27 & 6.31 & 6.27 & 6.27 & 6.27 & 6.54 & 6.46 & 6.38 & 6.27 & 6.54 \\
\hline Dual/poly & 6.09 & 6.13 & 6.00 & 6.03 & 6.00 & 6.22 & 6.09 & 6.03 & 6.09 & 6.22 \\
\hline \multicolumn{11}{|l|}{ Fetal harm } \\
\hline All & 6.17 & 6.21 & 6.19 & 6.17 & 6.19 & 6.28 & 6.19 & 6.22 & 6.21 & 6.28 \\
\hline WPT-only & 6.35 & 6.42 & 6.35 & 6.31 & 6.31 & 6.46 & 6.31 & 6.38 & 6.38 & 6.46 \\
\hline Dual/poly & 6.03 & 6.03 & 6.06 & 6.06 & 6.09 & 6.13 & 6.09 & 6.09 & 6.06 & 6.13 \\
\hline
\end{tabular}

Use groups are based on WPT use regardless of flavour ( $n=26$ WPT-only users; $n=32$ dual/poly WPT users). Preferences and perceptions were rated on a 1-7 scale with higher values indicating greater flavour preference or greater perceptions of the harmfulness. Bold font indicates statistically significant differences between WPT-only and dual/poly WPT users by Wilcoxon test.

${ }^{*} \mathrm{P} \leq 0.05$.

WPT, waterpipe tobacco.

higher $(\mathrm{ps}<0.05)$ than all flavours except fruit and candy; preferences for candy were higher $(\mathrm{ps}<0.05)$ than coffee, spice and tobacco flavours. Perceptions of harm to general health, pregnancy and fetal health did not differ among flavours ( $p s>0.950$ ). WPT-only users reported higher preferences (liking, attractiveness, pleasant, interest) for menthol/mint flavours (ps<0.05), while harm perceptions were uniformly elevated for WPT-only and dual/poly users for all three harm scales (general, pregnancy, fetal).

\section{Preferences and perceptions as predictors of flavoured WPT events}

Regression models investigating associations among preferences, harm perceptions and use of flavoured WPT are presented in table 3. Analyses were restricted to menthol/mint and fruit flavours because these flavours were used by $68 \%$ and $48 \%$ of participants in $68 \%$ and $14 \%$ of WPT events, respectively. Preferences for both menthol/mint and fruit-flavoured WPT were associated with more flavoured WPT use events across preconception and pregnancy (ps $\leq 0.007)$. WPT-only users and participants of Latina ethnicity reported greater menthol/mint flavoured WPT use events compared with non-Latina participants and dual/poly users, respectively (ps <0.001).

\section{Preferences and perceptions as predictors of biomarkers of exposure}

Regression models investigating associations among preferences, harm perceptions and biomarkers are presented in table 4 . These models predicted detection of cotinine, benzene and butadiene, given prior evidence for associations with WPT use. ${ }^{24}$ Higher preference scores for menthol/mint flavours were associated with $>2 \mathrm{x}$ increased likelihood of detection of cotinine or benzene (ps $\leq 0.038$ ); however, more menthol/mint flavoured WPT use events and higher perceptions of fetal harm from menthol/mint flavoured WPT were associated with decreased likelihood of cotinine and benzene detection (ps $\leq 0.038$ ). Finally, each additional fruit-flavoured WPT use event was associated with a nearly twofold increased likelihood of butadiene detection $(\mathrm{p}=0.038)$. No other statistically significant associations between flavoured WPT use events or harm perceptions and biomarkers were found (ps>0.05). Latina ethnicity was associated with increased detection of butadiene, but decreased cotinine detection ( $\mathrm{ps} \leq 0.043$ ).

\section{DISCUSSION}

We investigated use, preference and perceptions of WPT flavourings among pregnant women. Pregnant WPT users 


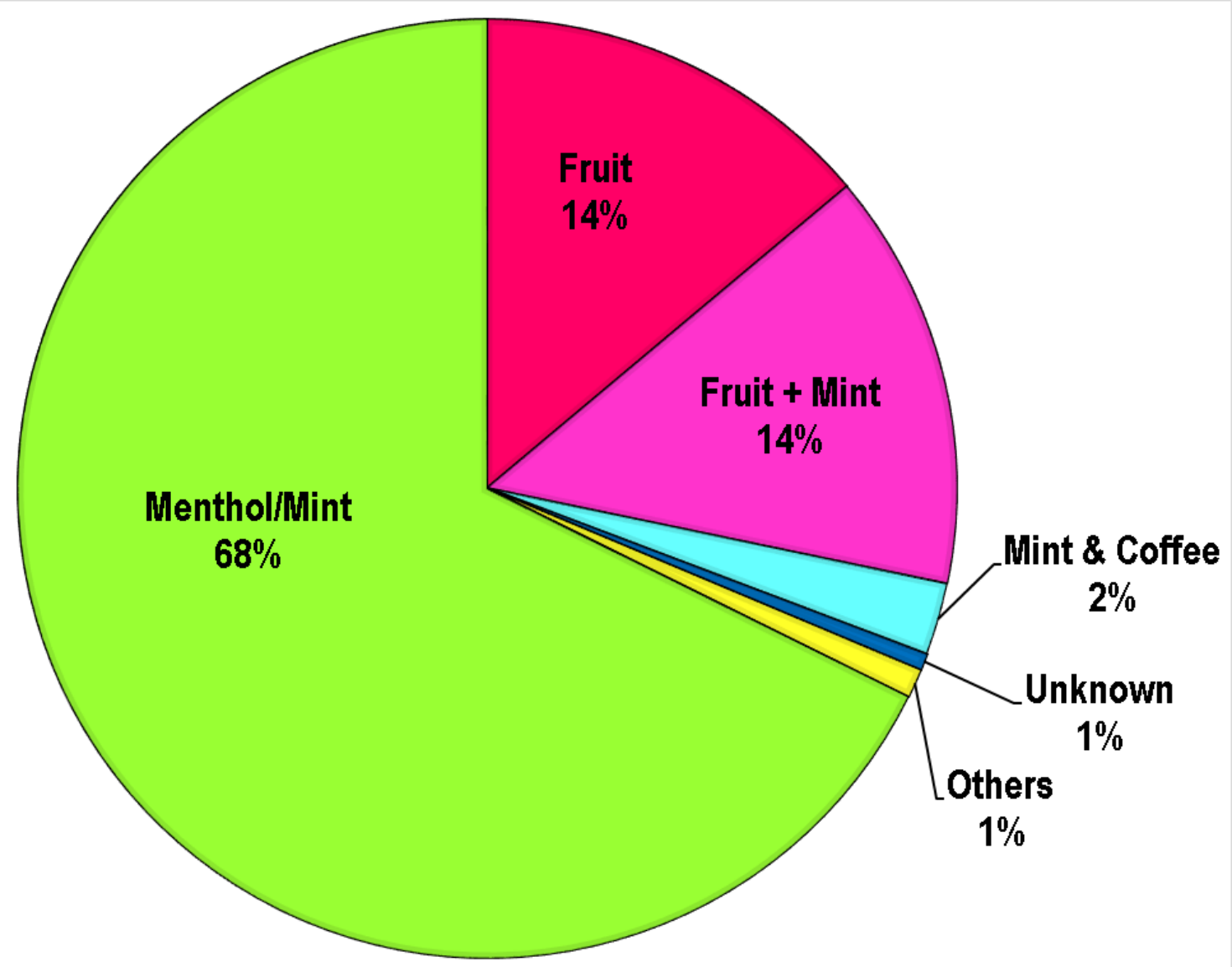

Figure 1 Proportion of flavours used across all WPT use events $(\mathrm{N}=1376)$ during preconception and pregnancy. Other flavours included: candy, fruit + candy, mint + chocolate, fruit + chocolate, coffee, alcohol, other beverages. WPT, waterpipe tobacco.

reported nearly exclusive use of flavoured tobacco; the majority of preconception/prenatal WPT use episodes involved menthol/ mint flavoured WPT, followed by fruit flavours. Preferences were highest for fruit, menthol/mint and sweet-flavoured WPT. Harm perceptions were high, but did not differ by flavour. Preferences

Table 3 Associations between flavour preferences, harm perceptions and use of flavoured WPT during preconception and pregnancy ( $\mathrm{N}=58)$

\begin{tabular}{|c|c|c|c|c|}
\hline \multirow[b]{2}{*}{ Predictor } & \multicolumn{2}{|l|}{ Menthol/Mint* } & \multicolumn{2}{|l|}{ Fruit $†$} \\
\hline & IRR (95\% Cl) & $P$ value & IRR (95\% Cl) & $P$ value \\
\hline Age & 0.98 (0.90 to 1.07$)$ & 0.723 & $1.08(0.97$ to 1.20$)$ & 0.152 \\
\hline Latina ethnicity $\ddagger$ & 4.01 (1.88 to 8.54$)$ & $<0.001$ & 0.74 (0.28 to 1.97$)$ & 0.546 \\
\hline Flavour preferences & 1.77 (1.39 to 2.25$)$ & $<0.001$ & 1.96 (1.20 to 3.19$)$ & 0.007 \\
\hline \multicolumn{5}{|l|}{ Flavour perceptions } \\
\hline General harm & 0.90 (0.67 to 1.22$)$ & 0.505 & 1.17 (0.85 to 1.62$)$ & 0.346 \\
\hline Fetal harm & 0.95 (0.73 to 1.26$)$ & 0.740 & 0.83 (0.58 to 1.19$)$ & 0.312 \\
\hline Dual/poly WPT use§ & $0.25(0.12$ to 0.52$)$ & $<0.001$ & $1.09(0.40$ to 2.93$)$ & 0.872 \\
\hline
\end{tabular}

Negative binomial regression with robust variance estimation was used for all count variables (menthol/mint and fruit-flavoured WPT events over preconception and first trimester). Age, preferences and perceptions (general harm, fetus) are continuous variables; ethnicity ( $1=$ Latina; $0=$ non-Latina) and use status ( $1=$ dual/poly WPT user; $0=$ WPT only user) were dummy coded.

Bold font indicates statistically significant IRRs.

*Overdispersion parameter: $\alpha=1.84$ (95\% Cl 1.27 to 2.66 ).

tOverdispersion parameter: $\alpha=2.70(95 \% \mathrm{Cl} 1.57$ to 4.64$)$.

$\ddagger$ Reference category is non-Latina (0).

$\S$ Reference category is WPT-only users (0).

IRR, incidence rate ratio; WPT, waterpipe tobacco. for fruit and menthol/mint flavours predicted preconception/ prenatal use of flavoured WPT, whereas harm perceptions did not. Compared with dual/poly WPT use, WPT-only users used more WPT, were more likely to use menthol/mint flavours, were less likely to use fruit flavours and had lower rates of detectable WPT/tobacco smoke exposure biomarkers.

High rates of use of mint and sweet flavours support our hypotheses and complement studies of WPT use and other tobacco product (ie, e-cigarette) use in non-pregnant populations. ${ }^{5863}$ However, in the present study, menthol/mint was used more frequently than fruit or other flavours-particularly among WPT-only users, whereas most prior studies with non-pregnant samples have shown greatest use of fruit flavours, with menthol/ mint used less frequently. For example, in a sample of $\sim 75$ 000 US adults, order of prevalence of WPT flavours use was: fruit (74\%), menthol/mint (19\%), and candy and other sweets $(17 \%){ }^{58}$ In prior studies of both pregnant and non-pregnant e-cigarette users, however, rates of use of fruit and menthol/mint flavoured e-cigarettes were similar. ${ }^{42} 5864-66$

Intriguingly, although pregnant women reported greater use of menthol/mint than fruit flavours, they endorsed similar preferences for both menthol/mint and fruit, followed by candy and other sweet flavours, which were preferred over coffee, spice and unflavoured WPT. Further, we found that greater preferences for both menthol/mint and fruit flavours predicted flavoured WPT use during preconception/pregnancy. Results complement findings from discrete-choice experiments, in which participants-especially women-were more likely to choose various fruit-over unflavoured WPT. ${ }^{67}{ }^{68}$ Results also complement 


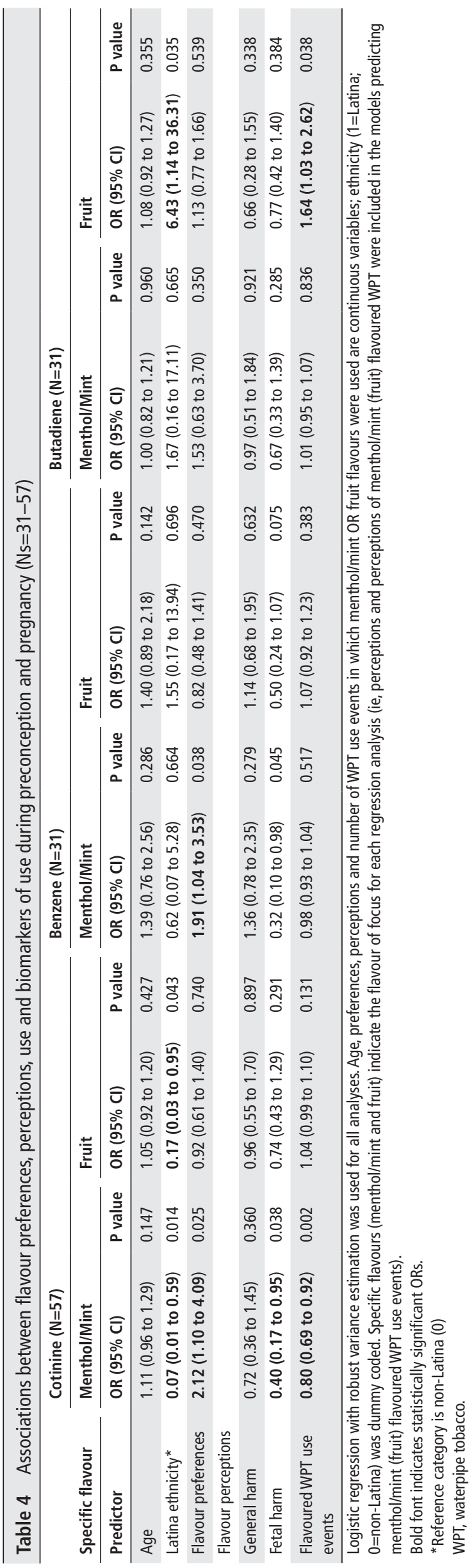

findings from studies of e-cigarette and other tobacco product flavour preferences in adults, young adults, youth and pregnant women. Sweet flavours were preferred among youth and young adult non-smokers and were associated with use and intentions to use, whereas menthol/mint was preferred among adults. ${ }^{64-66}$ In a study of e-cigarette flavour preferences among pregnant women, fruit and candy-flavoured e-cigarettes were preferred over menthol/mint flavours. ${ }^{42}$

Although preferences and use episodes differed by flavour, harm perceptions for general, pregnancy and fetal health did not. Perceptions of pregnancy and fetal harm were uniformly high (average of $>6.1$ on a 7-point scale) across all flavours (although fetal harm perceptions showed some evidence of variability despite high averages). Further, harm perceptions showed no associations with episodes of (flavoured) preconception/prenatal WPT use. Prior WPT studies have shown decreased harm perceptions for (flavoured) WPT versus cigarettes, but have not investigated harm perceptions across WPT flavours. ${ }^{364}$ However, prior studies of e-cigarette flavourings revealed decreased harm perceptions for fruit and sweet-flavours versus tobacco flavours. ${ }^{66}$ Results from this study extend prior research by our group using similar methods to the present study - one in pregnant women focused on e-cigarettes and one in reproductive-age women focused on WPT. In both studies, harm perceptions did not differ by flavour and were not associated with e-cigarette/WPT use. ${ }^{42}{ }^{43}$ Lack of differences in harm perceptions across flavours may relate to our focus on pregnant women and widespread public health and education campaigns regarding the harmful effects of tobacco use during pregnancy.

To our knowledge, the present study is the first to measure biomarkers of WPT/tobacco smoke exposure in pregnant WPT users. Although overall levels of exposure biomarkers were low, preference for menthol/mint flavoured WPT was associated with double the likelihood of detection of cotinine or benzene. Further, use of fruit flavours was associated with increased likelihood of butadiene detection, while use of menthol/mint flavours was associated with decreased cotinine detection. In addition, consistent with prior literature, dual/poly WPT users showed higher levels of cotinine and certain VOCs vs WPT-only users. Results should be interpreted with caution given the smaller sample size for biomarker analyses and because biomarkers were assessed at the time of the interview, not in relation to recent episodes of WPT use. Given the sporadic nature of WPT use for most participants and the relatively short elimination half-life of the biomarkers measured (hours), the present study likely underestimates maternal/fetal exposure to toxic chemicals.

\section{Regulatory implications}

As regulatory policies regarding WPT are formulated in the USA and other countries, ${ }^{3869}$ it may be relevant to consider pregnant women as a uniquely vulnerable population. Labelling requirements for WPT and devices, and warnings in WPT establishments advising pregnant women not to use WPT are warranted immediately. ${ }^{202140} 70$ Education for prenatal providers regarding how to screen and counsel women who use WPT during pregnancy is also needed.

Our study highlights the importance of comprehensive flavoured tobacco product regulation. To date, in the USA, only a handful of municipalities in California have banned the sale of all flavours in all tobacco products, ${ }^{71} 72$ and no states have done so, although the California legislature is currently considering a proposal to end flavoured tobacco sales statewide (Senate Bill 38). ${ }^{73}$ Outside the USA, Canada has taken the lead 
in regulating flavoured tobacco products, with five provinces/ territories (Ontario, Quebec, New Brunswick, Prince Edward Island and Newfoundland and Labrador) adopting flavoured tobacco bans that include WPT. ${ }^{74}$ Continued research on WPT use, preferences and biomarkers during pregnancy and in reproductive-age women may inform the development of regulatory policies regarding WPT flavours. ${ }^{75}$ Research on the unique impact of flavourings, WPT and flavoured WPT on human fetal development, which is urgently needed, ${ }^{76}$ may also play a role in influencing the regulatory approach to the continued sale of flavoured WPT.

\section{Limitations}

Several limitations should be considered when interpreting our findings. First, we analysed baseline data from our first cohort in an ongoing longitudinal study. Although we used TLFB methods to cue recall, all data were collected from a single interview and thus cannot support causal inferences. Second, our study sampled a small $(\mathrm{N}=58)$ convenience sample of low-income, racially/ethnically diverse pregnant women from southern New England (Rhode Island and Massachusetts). Selection bias is thus possible, and results may not generalise to the local population or other USA regions or countries. Further, including only English-speaking women may have limited the generalisability of findings related to Latina ethnicity. Inclusion of women who were recruited by both active (approached in clinic) and passive (advertisements) means may also limit the generalisability of the sample. Nationally, internationally or locally representative studies with larger samples are needed to better elucidate WPT/flavours use and preferences in pregnancy. Finally, we assessed use and preference of multiple broad categories of WPT flavours (eg, fruit) but did not assess specific flavours (eg, apple). Future research should investigate specific flavours that may be appealing to pregnant mothers.

\section{What this paper adds}

What is already known on this subject?

- Waterpipe (hookah) tobacco (WPT) use is a growing public health problem among pregnant and reproductive-age women.

- Given known impacts of prenatal tobacco use on maternal and infant morbidity, pregnant WPT users represent a uniquely vulnerable population.

- Sweet flavours contribute to the appeal of WPT and are a potential regulatory target.

What important gaps in knowledge exist on this topic?

- Little research has addressed WPT use in pregnancy.

- Little is known regarding characteristics of WPT use by pregnant women that are amenable to regulatory efforts to protect the health of women and children.

What this paper adds?

- Pregnant WPT users reported greatest use of and preferences for menthol/mint and fruit flavours.

- Preferences for menthol/mint and fruit flavoured WPT predicted flavoured WPT use and biomarkers of exposure.

- Findings in this vulnerable population highlight the need to regulate both sweet and mint/menthol flavours in WPT.

\section{CONCLUSIONS}

Given increasing use of WPT by pregnant and reproductive-age women and known maternal and fetal toxicity of tobacco, it is critical to understand characteristics of WPT that are associated with maternal use and are amenable to regulation. In this study, pregnant WPT users reported nearly exclusive use of flavoured WPT, with menthol/mint and fruit-flavoured WPT used most frequently and most preferred. Further, preferences for fruit and menthol/mint flavours were associated with greater WPT use and exposure biomarkers despite high levels of harm perception for all flavours. Results from this uniquely vulnerable population highlight the need to implement warning labels for pregnant women on WPT, devices and WPT establishments, and regulate sweet and mint/menthol WPT flavours.

Acknowledgements We gratefully acknowledge the women who contributed to this study. We are also grateful to the Maternal-Infant Studies Laboratory staff for their assistance with data collection. We thank Pamela Borek for administrative assistance with this manuscript.

Contributors LS designed and conceptualised the study, coordinated the acquisition of data, drafted the initial manuscript, contributed to the interpretation of results and revised the manuscript critically for important intellectual content. EW provided substantial contribution to recruitment and data collection, and in revising the manuscript critically for intellectual content. KM provided substantial contribution to recruitment and data collection, and in revising the manuscript critically for intellectual content. MC provided input into the study design, and in revising the manuscript critically for intellectual content. GSH provided input into study design and in revising the manuscript critically for intellectual content. TE provided input into study design and in revising the manuscript critically for intellectual content. LAJS-S designed and conceptualised the study, conducted statistical analyses and drafted the results, and revised the manuscript critically for important intellectual content. All authors approved the final manuscript as submitted and agree to be accountable for all aspects of the work.

Funding Funding for this study and manuscript preparation was supported by the National Institute on Drug Abuse (NIDA) of the National Institutes of Health and the Center for Tobacco Products of the US Food and Drug Administration (FDA) under grant 5R01 DA042484 to LS and LAJS-S. TE is also supported by NIDA under Award Number U54 DA036105 and the Center for Tobacco Products of the FDA

Disclaimer The content is solely the responsibility of the authors and does not necessarily represent the official views of the National Institutes of Health or the Food and Drug Administration. NIDA, CTP and FDA had no role in the study design, collection, analysis or interpretation of the data, writing the manuscript, or the decision to submit the paper for publication.

Competing interests TE is a paid consultant in litigation against the tobacco industry and is named on a patent for a device that measures the puffing behaviour of electronic cigarette users.

Patient consent for publication Not required.

Ethics approval All study procedures were approved by local institutional review boards.

Provenance and peer review Not commissioned; externally peer reviewed. Data availability statement Data are available on reasonable request.

ORCID iD

Laura Stroud http://orcid.org/0000-0002-2138-968X

\section{REFERENCES}

1 U.S. Department of Health and Human Services. The health consequences of Smoking - 50 years of progress: a report of the surgeon General; U.S. department of health and Human services, centers for disease control and prevention, National center for chronic disease prevention and health promotion, office on smoking and health. Atlanta, GA, 2014

2 Villanti AC, Cobb CO, Cohn AM, et al. Correlates of hookah use and predictors of hookah trial in U.S. young adults. Am J Prev Med 2015:48:742-6.

3 Richardson A, Williams V, Rath J, et al. The next generation of users: prevalence and longitudinal patterns of tobacco use among US young adults. Am J Public Health 2014;104:1429-36.

4 Cohn AM, Johnson AL, Rath JM, et al. Patterns of the co-use of alcohol, marijuana, and emerging tobacco products in a national sample of young adults. Am J Addict 2016;25:634-40 
5 Jawad M, Charide R, Waziry R, et al. The prevalence and trends of waterpipe tobacco smoking: a systematic review. PLoS One 2018;13:e0192191.

6 Agaku IT, King BA, Husten CG, et al. Tobacco product use among adults--United States, 2012-2013. MMWR Morb Mortal Wkly Rep 2014;63:542-7.

7 Kasza KA, Ambrose BK, Conway KP, et al. Tobacco-Product use by adults and youths in the United States in 2013 and 2014. N Engl J Med 2017;376:342-53.

8 Dar-Odeh NS, Abu-Hammad OA. The changing trends in tobacco smoking for young Arab women; narghile, an old habit with a liberal attitude. Harm Reduct J 2011;8.

9 Maziak W, Taleb ZB, Bahelah R, et al. The global epidemiology of waterpipe smoking. Tob Control 2015;24 Suppl 1(Suppl 1):i3-12.

10 Jawad M, Abdulrahim S, Daouk A. The social patterning of tobacco use among women in Jordan: the protective effect of education on cigarette smoking and the deleterious effect of wealth on cigarette and waterpipe smoking. Nicotine Tob Res 2016:18:379-85.

11 Kurti AN, Redner R, Lopez AA, et al. Tobacco and nicotine delivery product use in a national sample of pregnant women. Prev Med 2017;104:50-6.

12 U.S. Department of Health and Human Services. The health consequences of smoking: a report of the surgeon General. Atlanta, GA: U.S. department of health and Human services, centers for disease control and prevention, National center for chronic disease prevention and health promotion, office on smoking and health, 2004.

13 Chaaya M, Awwad J, Campbell OMR, et al. Demographic and psychosocial profile of smoking among pregnant women in Lebanon: public health implications. Matern Child Health J 2003;7:179-86.

14 Azab M, Khabour OF, Alzoubi KH, et al. Exposure of pregnant women to waterpipe and cigarette smoke. Nicotine Tob Res 2013;15:231-7.

15 Chaaya M, Jabbour S, El-Roueiheb Z, et al. Knowledge, attitudes, and practices of argileh (water pipe or hubble-bubble) and cigarette smoking among pregnant women in Lebanon. Addict Behav 2004:29:1821-31.

16 Mirahmadizadeh A, Nakhaee N. Prevalence of waterpipe smoking among rural pregnant women in southern Iran. Med Princ Pract 2008;17:435-9.

17 AkI EA, Gunukula SK, Aleem S, et al. The prevalence of waterpipe tobacco smoking among the general and specific populations: a systematic review. BMC Public Health 2011;11.

18 Kathirvel S, Thakur IS, Sharma S. Women and tobacco: a cross sectional study from North India. Indian J Cancer 2014;51 Suppl 1(Suppl 1):S78-S82.

19 Kassem NOF, Kassem NO, Jackson SR, et al. Benzene uptake in Hookah smokers and non-smokers attending Hookah social events: regulatory implications. Cancer Epidemiol Biomarkers Prev 2014;23:2793-809.

20 Eissenberg T, Shihadeh A. Waterpipe tobacco and cigarette smoking: direct comparison of toxicant exposure. Am J Prev Med 2009;37:518-23.

21 Cobb CO, Shihadeh A, Weaver MF, et al. Waterpipe tobacco smoking and cigarette smoking: a direct comparison of toxicant exposure and subjective effects. Nicotine Tob Res 2011;13:78-87.

22 Hecht SS. Tobacco carcinogens, their biomarkers and tobacco-induced cancer. Nat Rev Cancer 2003:3:733-44.

23 Jacob P, Abu Raddaha AH, Dempsey D, et al. Comparison of nicotine and carcinogen exposure with water pipe and cigarette smoking. Cancer Epidemiol Biomarkers Prev 2013:22:765-72.

24 St Helen G, Benowitz NL, Dains KM, et al. Nicotine and carcinogen exposure after water pipe smoking in hookah bars. Cancer Epidemiol Biomarkers Prev 2014;23:1055-66

25 Hoffmann D, Brunnemann KD, Hoffmann I. Significance of benzene in tobacco carcinogenesis. Princeton Scientific Publishing Co: Advances in Modern Environmental Toxicology Benzene: Occupational and Environmental Hazards-Scientific Update Princeton, 1989

26 Waziry R, Jawad M, Ballout RA, et al. The effects of waterpipe tobacco smoking on health outcomes: an updated systematic review and meta-analysis. Int J Epidemiol 2017:46:32-43

27 Haddad L, Kelly DL, Weglicki LS, et al. A systematic review of effects of waterpipe smoking on cardiovascular and respiratory health outcomes. Tob Use Insights 2016;9:TUI.S39873-28.

28 Awan KH, Siddiqi K, Patil S, et al. Assessing the Effect of Waterpipe Smoking on Cancer Outcome - a Systematic Review of Current Evidence. Asian Pac J Cancer Prev 2017:18:495-502.

29 El-Zaatari ZM, Chami HA, Zaatari GS. Health effects associated with waterpipe smoking. Tob Control 2015;24 Suppl 1(Suppl 1):i31-43.

30 Tamim H, Yunis KA, Chemaitelly H, et al. Effect of narghile and cigarette smoking on newborn birthweight. BJOG 2008;115:91-7.

31 Nuwayhid IA, Yamout B, Azar G, et al. Narghile (hubble-bubble) smoking, low birth weight, and other pregnancy outcomes. Am J Epidemiol 1998;148:375-83.

32 Oudgenoeg-Paz O, Mulder H, Jongmans MJ, et al. The link between motor and cognitive development in children born preterm and/or with low birth weight: a review of current evidence. Neurosci Biobehav Rev 2017:80:382-93.

33 Hallit S, Raherison C, Waked M, et al. Association between caregiver exposure to Toxics during pregnancy and childhood-onset asthma: a case-control study. Iran J Allergy Asthma Immunol 2017;16:488-500.
34 AkI EA, Ward KD, Bteddini D, et al. The allure of the waterpipe: a narrative review of factors affecting the epidemic rise in waterpipe smoking among young persons globally. Tob Control 2015;24 Suppl 1(Suppl 1):i13-21.

35 Eshah NF, Froelicher ES, Knowledge FES. Knowledge, attitudes, beliefs and patterns of waterpipe use among Jordanian adults who exclusively smoke waterpipes. Eur J Cardiovasc Nurs 2018;17:85-92.

36 Kowitt S, Meernik C, Baker H, et al. Perceptions and experiences with flavored NonMenthol tobacco products: a systematic review of qualitative studies. Int I Environ Res Public Health 2017;14.

37 Jawad M, El Kadi L, Mugharbil S, et al. Waterpipe tobacco smoking legislation and policy enactment: a global analysis. Tob Control 2015;24 Suppl 1(Suppl 1):i60-5.

38 U.S. Food and Drug Administration. Deeming tobacco products to be subject to the federal food, drug, and cosmetic Act, as amended by the family smoking prevention and tobacco control act; restrictions on the sale and distribution of tobacco products and required warning statements for tobacco products. final rule. (FDA-2014-N-0189) silver spring, MD, 2016. Available: https://www. federalregister.gov/documents/2016/05/10/2016-10685/deeming-tobaccoproducts-to-be-subject-to-the-federal-food-drug-and-cosmetic-act-as-amendedby-the

39 Cobb C, Ward KD, Maziak W, et al. Waterpipe tobacco smoking: an emerging health crisis in the United States. Am J Health Behav 2010;34:275-85.

40 Shihadeh A, Schubert J, Klaiany J, et al. Toxicant content, physical properties and biological activity of waterpipe tobacco smoke and its tobacco-free alternatives. Tob Control 2015;24 Suppl 1(Suppl 1):i22-30.

41 Faas MM, Melgert BN, de Vos P. A brief review on how pregnancy and sex hormones interfere with taste and food intake. Chemosens Percept 2010;3:51-6.

42 Stroud LR, Papandonatos GD, Borba K, et al. Flavored electronic cigarette use, preferences, and perceptions in pregnant mothers: a correspondence analysis approach. Addict Behav 2019:91.

43 Scott-Sheldon LAJ, Stroud LR. Preferences and perceptions of flavored Hookah tobacco among US women. Am J Health Behav 2018:42:37-46.

44 Sobell LC, Sobell MB. Timeline Followback: A technique for assessing self-reported alcohol consumption. In: Litten R, Allen J, eds. Measuring alcohol consumption: psychosocial and biochemical methods. New Jersey: Humana Press, 1992.

45 Sobell LC, Sobell MB. Alcohol Timeline Followback users' manual. Toronto, Canada: Addiction Research Foundation, 1995.

46 Robinson SM, Sobell LC, Sobell MB, et al. Reliability of the Timeline Followback for cocaine, cannabis, and cigarette use. Psychol Addict Behav 2014;28:154-62.

47 Scott-Sheldon LAJ, Stroud LR. The tobacco flavors interview. Providence, RI: The Miriam Hospital, Centers for Behavioral and Preventive Medicine, 2015.

48 U.S. Department of Health and Human Services. Population Assessment of Tobacco and Health (PATH) Study 2013-2016 [United States] Restricted-Use Files. InterUniversity Consortium for Political and Social Research, 2016.

49 Baeza-Loya S, Viswanath H, Carter A, et al. Perceptions about e-cigarette safety may lead to e-smoking during pregnancy. Bull Menninger Clin 2014;78:243-52.

50 Popova L, Ling PM. Perceptions of relative risk of snus and cigarettes among US smokers. Am J Public Health 2013;103:e21-3.

51 Jarvis MJ, Tunstall-Pedoe $\mathrm{H}$, Feyerabend C, et al. Comparison of tests used to distinguish smokers from nonsmokers. Am J Public Health 1987;77:1435-8.

52 Jacob P, Wilson M, Benowitz NL. Improved gas chromatographic method for the determination of nicotine and cotinine in biologic fluids. J Chromatogr B Biomed SC App/ 1981:222:61-70.

53 Jacob P, Yu L, Wilson M, et al. Selected ion monitoring method for determination of nicotine, cotinine and deuterium-labeled analogs: absence of an isotope effect in the clearance of (S)-nicotine-3', 3'-d2 in humans. Biol Mass Spectrom 1991;20:247-52.

54 Sandberg A, Sköld CM, Grunewald J, et al. Assessing recent smoking status by measuring exhaled carbon monoxide levels. PLoS One 2011;6:e28864.

55 StataCorp. Stata statistical software release 15. College Station, TX: Stata Corp, LLC, 2017.

56 Hornung RW, Reed LD. Estimation of average concentration in the presence of Nondetectable values. Appl Occup Environ Hyg 1990;5:46-51.

57 Barr DB, Wilder LC, Caudill SP, et al. Urinary creatinine concentrations in the U.S. population: implications for urinary biologic monitoring measurements. Environ Health Perspect 2005;113:192-200.

58 Bonhomme MG, Holder-Hayes E, Ambrose BK, et al. Flavoured non-cigarette tobacco product use among US adults: 2013-2014. Tob Control 2016;25(Suppl 2):ii4-13.

59 Hukkanen J, Jacob P, Benowitz NL. Metabolism and disposition kinetics of nicotine. Pharmacol Rev 2005;57:79-115.

60 Grosse Y, Baan R, Straif K, et al. Carcinogenicity of 1,3-butadiene, ethylene oxide, vinyl chloride, vinyl fluoride, and vinyl bromide. Lancet Oncol 2007;8:679-80.

61 United States department of health and Human services, centers for disease control and prevention, National center for health statistics, division of vital statistics. Natality Public-Use data, 2007-2017, 2018. Available: http://wonder.cdc.gov/natality-current. html [Accessed 1 Apr 2019].

62 Cropsey KL, Trent LR, Clark CB, et al. How low should you go? determining the optimal cutoff for exhaled carbon monoxide to confirm smoking abstinence when using cotinine as reference. Nicotine Tob Res 2014;16:1348-55. 
63 Villanti AC, Johnson AL, Ambrose BK, et al. Flavored tobacco product use in youth and adults: findings from the first wave of the path study (2013-2014). Am J Prev Med 2017;53:139-51.

64 Huang L-L, Baker HM, Meernik C, et al. Impact of non-menthol flavours in tobacco products on perceptions and use among youth, young adults and adults: a systematic review. Tob Control 2017;26:709-19.

65 Hoffman AC, Salgado RV, Dresler C, et al. Flavour preferences in youth versus adults: a review. Tob Control 2016;25(Suppl 2):ii32-9.

66 Zare S, Nemati M, Zheng Y. A systematic review of consumer preference for e-cigarette attributes: flavor, nicotine strength, and type. PLoS One 2018;13:e0194145.

67 Salloum RG, Maziak W, Hammond D, et al. Eliciting preferences for waterpipe tobacco smoking using a discrete choice experiment: implications for product regulation. BMJ Open 2015;5:e009497.

68 Salloum RG, Nakkash R, Abu-Rmeileh NME, et al. Individual-level determinants of waterpipe smoking demand in four Eastern-Mediterranean countries. Health Promot Int 2018;24.

69 eWHO Study Group on Tobacco Product Regulation (TobREg). Advisory not. Waterpipe tobacco smoking: health effects, research needs and recommended actions for regulators. Geneva: World Health Organization, 2015.
70 Primack BA, Carroll MV, Weiss PM, et al. Systematic review and meta-analysis of inhaled toxicants from waterpipe and cigarette smoking. Public Health Rep 2016;131:76-85.

71 Clift T. Sacramento City Council approves ban on sale of flavored tobacco products. The Sacramento Bee, 2019.

72 San Francisco Department of Public Health. San Francisco's flavored tobacco sales ban is now in effect! 2018. Available: https://www.sfdph.org/dph/files/EHSdocs/Tobacco/ SFDPHFlavoredTobaccoFactSheet.pdf [Accessed 15 Apr 2019].

73 Flavored tobacco products, S. 22995, California legislature, 2019-2029 regular session Sess 2018.

74 Canadian Cancer Society. Overview summary of Federal/Provincial/Territorial tobacco control legislation in Canada, 2017. Available: http://convio.cancer.cal documents/Legislative_Overview-Tobacco_Control-F-P-T-2017-final.pdf [Accessed 15 Apr 2019].

75 U.S. Food and Drug Administration. Advance notice of proposed rulemaking regulation of flavors in tobacco products. Federal Register 2018;83:12294-301.

76 Bahl V, Lin S, Xu N, et al. Comparison of electronic cigarette refill fluid cytotoxicity using embryonic and adult models. Reprod Toxicol 2012;34:529-37. 